\title{
Miniaturized Drug Delivery System for Biomedical Applications
}

\author{
Khalil Moussi, Mohammed AlDajani, Jurgen Kosel \\ Computer, Electrical and Mathematical Sciences and Engineering Division \\ King Abdullah University of Science and Technology \\ Thuwal, Saudi Arabia \\ E-mails: jurgen.kosel@kaust.edu.sa,khalil.moussi@kaust.edu.sa
}

\begin{abstract}
A miniaturized 3D printed drug delivery device powered wirelessly is presented. The device is composed of an electrochemical micropump, a 3D printed reservoir with microneedles, and a wireless powering unit. The electrochemical pump features an expandable (up to $300 \%$ ) Parylene $\mathrm{C}$ microbellows membrane fabricated by mold casting, using a twophoton polymerization 3D printing technique. The hollow microneedles are $100 \mu \mathrm{m}$ in diameter and $300 \mu \mathrm{m}$ long. The fabrication process offers customizable device properties, where the shape, size, and performance can be tailored to fit a wide range of in vivo drug delivery applications. Delivery of $3.8 \pm 0.3$ $\mu \mathrm{L}$ within 10 seconds of actuation is demonstrated, using inductive wireless powering at a distance of $10 \mathrm{~mm}$ between the primary and secondary coils.
\end{abstract}

Keywords—drug delivery, micropump, microneedles, 3D printed

\section{INTRODUCTION}

Conventional drug delivery methods tend to show severe drawbacks in the efficacy of treatment, due to the difficulties in administrating the optimal drug dosage to the optimal tissue site with optimal frequency. Therefore, efforts have been concentrating on optimizing these critical parameters [1].

As an alternative to conventional techniques, implantable drug delivery systems have been a principal focus of development for the parenteral and oral drug administration, where extensive work has been done to optimize drug efficacy via localized delivery and dosage control providing new ways for accomplishing targeted therapeutic effects [1-2]. However, there remain opportunities for advancement by introducing novel microtechnologies in fabrication methods or engineering discoveries in concept realization, making such devices more versatile and effective [3]. In particular, microdevices based on electrolytic pumps have been greatly explored in the past [4-6]. These systems demonstrate the advantages of low power consumption, adequate driving force, and accurate flow control [7-8].

Bellow membranes are suitable as actuators for microelectrolytic pumps, due to their fitting pressure ranges [9]. The predictable performance and expansion profile of microbellows translates to controlled dosage in drug delivery. Due to micro-bellows' malleability, they easily expand under internal pressure, making them ideal membranes in electrolytic pumps [6-8]. Combined with Parylene C's biocompatibility, they act as a diaphragm isolating the drug reservoir from the pumping source preventing degradation and $\mathrm{pH}$ changes from water exposure [6]. Due to these pumps' minimal power requirements, wireless inductive powering units can be installed to achieve wireless actuation [10-11]. With an electromagnetic field from a transmitting coil, a current is induced in the receiving coil driving the electrolytic reaction in the pump. Past works have applied similar concepts, reporting drug delivery systems integrated with electrochemical micro pumps [6-8], [10-11].

Traditional fabrication and design methods used to limit the pump to be operated in very specific scenarios. With additive manufacturing methods such as two-photon polymerization, rigid applications can be avoided [12]. Following the same process, with minimal dimensional design edits, a set of versatile pumps can be fabricated and implanted at different sites, paving the way towards novel therapeutic options. Combined with the use of microneedles, these integrated systems have a promising potential for targeted drug delivery for the treatment of tumors and critical diseases like atherosclerosis. For such applications, the drug volume to be delivered ranges from 0.6 to $15 \mu \mathrm{L}$ [13-14]. By employing a flexible design and fabrication method, significant progress can be made finding the optimal compromise between device size, drug dosage (volume and rate), and power consumption.

In this study, a miniaturized and wirelessly powered drug delivery system is presented. The system includes an inductive powering unit, interdigitated electrodes for electrolysis, a Parylene $\mathrm{C}$ micro-bellows membrane [9], and a 3D printed reservoir with microneedles. Utilizing the tools and components mentioned, the presented design displays some advantages in size, powering, integration ease, versatility, and flexibility. The remaining of this manuscript is arranged as follows; section II presents the prototype design principle and fabrication methods, while section III describes and analyzes experimental data and simulations, and section IV concludes with key results.

\section{CONCEPT AND FABRICATION}

The drug delivery system is composed of three main parts; a 3D printed reservoir with microneedles, an electrolysis micropump, and an inductive powering unit, as shown in 

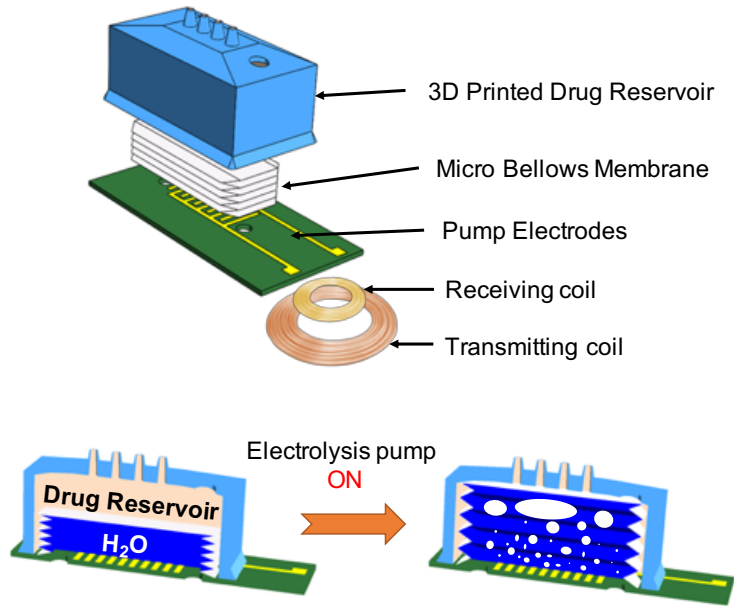

Fig. 1. Schematic diagram of the drug delivery system and operation concept. (a) 3D Design
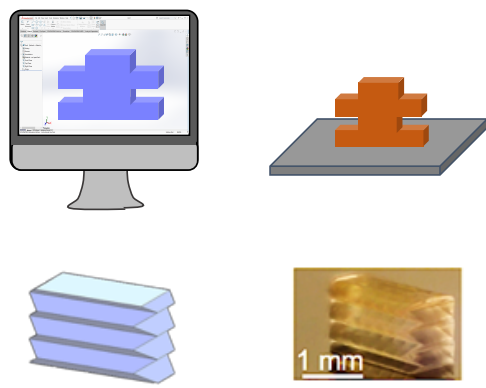

(c) PDMS casting
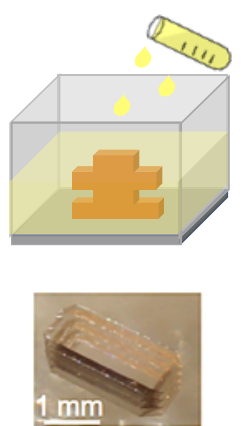

Silicon

IP-S photoresist

Fig. 3. Fabrication process of micro-bellows membrane.

Fig. 1. The pumping mechanism is based on the inflation of a Parylene $\mathrm{C}$ micro-bellows membrane, due to gas bubbles generated from the water electrolysis reaction. Such an actuation mechanism is distinguished over other methods with a significant volume change even in a pressurized medium and low power consumption [6], [15]. First, the micro-bellows membrane was assembled on top of the interdigitated electrodes, and about $1.5 \mu \mathrm{L}$ of $1 \mathrm{wt} \% \mathrm{NaCl}$ solution in DI water was injected inside the membrane. Then, the 3D printed reservoir was assembled on top of the electrochemical pump and filled with the liquid drug through a refill port. The transmitter unit was composed of wireless transmission modules (XKT412 and XKT335, Shenzhen Core Ketai Electronics Co., Ltd., Shenzhen, China) and a transmitting coil (33 mm outer diameter, $5 \mathrm{~mm}$ inner diameter and $1 \mathrm{~mm}$ thickness). Powered with a DC voltage generator $(5 \mathrm{~V}, 0.1 \mathrm{~A})$, the transmitter unit provided an output voltage and current of $\sim 10 \mathrm{~mA}$ and $5 \mathrm{~V}$, respectively, in the receiver's terminal (21 $\mathrm{mm}$ outer diameter, $10 \mathrm{~mm}$ inner diameter and $0.5 \mathrm{~mm}$ thickness) at $10 \mathrm{~mm}$ distance. When connecting the receiver terminal to the interdigitated electrodes, water electrolysis

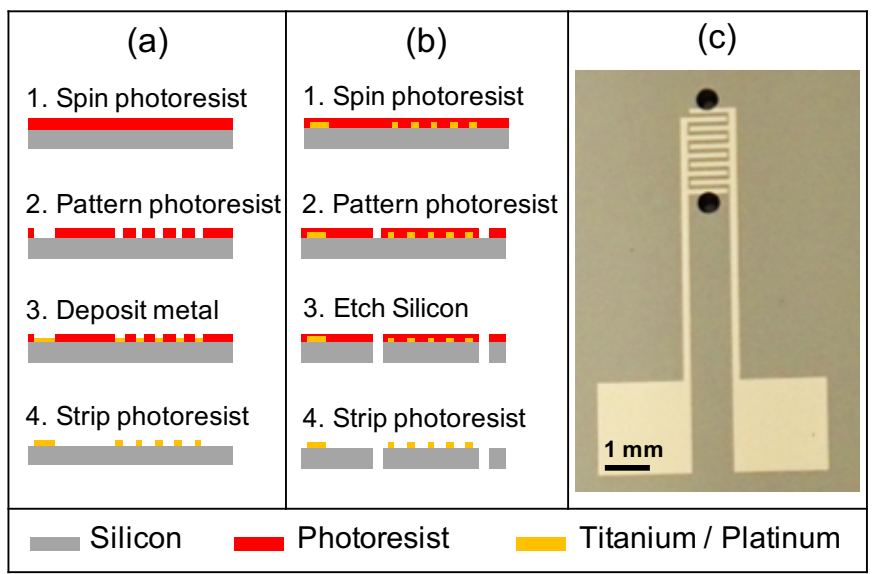

Fig. 2. Interdigitated electrodes fabrication process. (a) Patterning electrodes. (b) Etching holes. (c) Photograph of the final sample. (d) Sacrificial mold
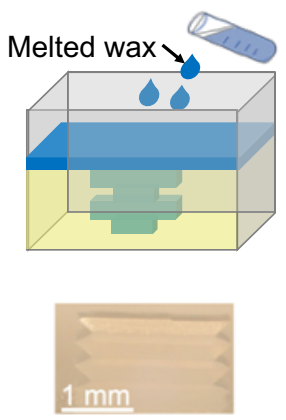

PDMS
(e) Parylene C coating
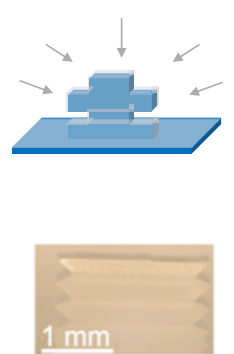

(f) Membrane release initiates and oxygen/hydrogen bubbles are produced. Consequently, the bellows membrane is inflated pushing the drug through the microneedles.

\section{A. Micropump fabrication}

Interdigitated electrodes made of 5 finger pairs $(100 \mu \mathrm{m} /$ $100 \mu \mathrm{m}$ elements width/spacing) with a total area of $1.25 \mathrm{~mm}^{2}$ were fabricated on silicon substrate, as shown in Fig. 2. A liftoff process using AZ ECI 3027 (MicroChemicals GmbH, Ulm, Germany) photoresist was employed to pattern the Ti/Pt $(30 \mathrm{~nm} / 300 \mathrm{~nm})$ electrodes (Fig. 2a). Two holes $(300 \mu \mathrm{m}$ in diameter) were created through the silicon substrate by Deep Reactive Ion Etching following the process described in Fig. 2b using the photoresist AZ 9260 (MicroChemicals GmbH, Ulm, Germany). These holes served to inject the electrolyte solution (1 wt $\% \mathrm{NaCl}$ solution in DI water) between the interdigitated electrodes and micro-bellows membrane.

The fabrication process of the Parylene $\mathrm{C}$ micro-bellows membrane summarized in Fig. 3 was previously reported [9]. The membrane's dimensions were $3 \mathrm{~mm}$ by $1.2 \mathrm{~mm}$ in length and width, respectively, with a height varying from $0.5 \mathrm{~mm}$, 
(a)

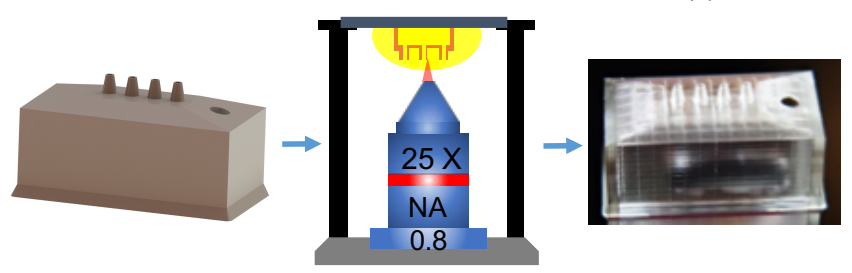

Fig. 4. 3D printed microchip fabrication process. (a) Design. (b) 3D printing. (c) Photograph of the 3D printed reservoir.

when the membrane is fully folded (deflated), to about $2 \mathrm{~mm}$, when it is fully expended (inflated). This large deflection is facilitated by five outlying triangular corrugations $(250 \mu \mathrm{m}$ of corrugation depth and length).

\section{B. 3D printed drug reservoir with microneedles}

The reservoir $(3.9 \mathrm{~mm} \times 2.1 \mathrm{~mm} \times 2 \mathrm{~mm})$ features 4 microneedles of $100 \mu \mathrm{m}$ in diameter and $300 \mu \mathrm{m}$ in length. The structure was 3D printed using a two-photon polymerization 3D printer (Photonic Professional GT, Nanoscribe GmbH, Germany) on a $25 \mathrm{~mm} \times 25 \mathrm{~mm}$ silicon substrate. A hole (1 $\mathrm{mm}$ in diameter) was etched in the center of the silicon substrate using a 50 Watts fiber laser (PLS6MW, Universal Laser Systems GmbH, Vienna, Austria) with a 1.06 $\mu \mathrm{m}$ wavelength, to facilitate the photoresist removal after $3 \mathrm{D}$ printing the reservoir structure. Before $3 \mathrm{D}$ printing, the silicon substrate was cleaned with acetone followed by isopropyl alcohol then it was rinsed with DI water and dried with a gentle stream of nitrogen gas. IP-S photoresist (IP-S, Nanoscribe GmbH, Germany) was then drop-cast. The 3D printer objective lens $(25 \times$ magnifications, NA 0.8$)$ was immersed in the resin, and the designed reservoir structure was printed layer-by-layer ( $2 \mu \mathrm{m}$ slicing distance) in a dip-in laser lithography configuration, (Fig. 4). Then, the structure (reservoir and microneedles) was developed by immersion in mr-DEV 600 (micro resist technology $\mathrm{GmbH}$, Germany) to

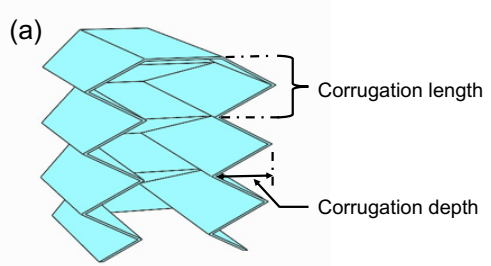

(b)

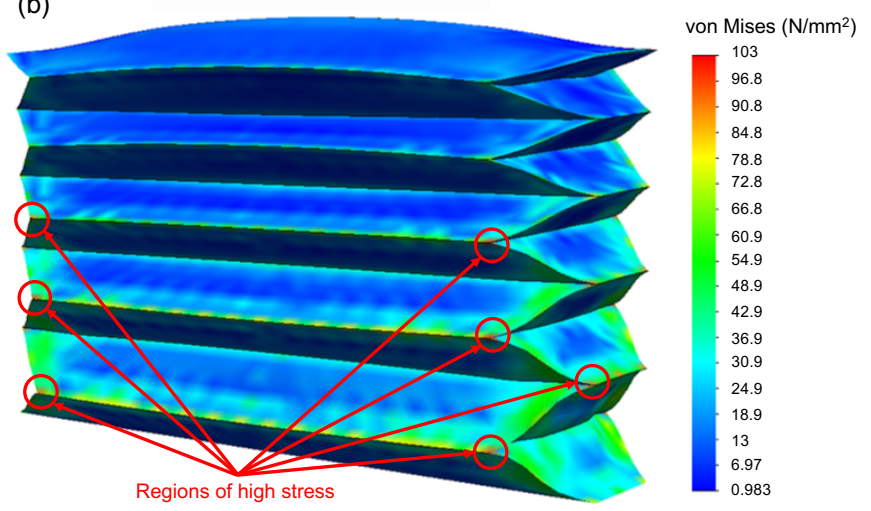

Fig. 5. (a) Schematic of the micro-bellows membrane parameters. (b) Finite element modeling of the Parylene $\mathrm{C}$ micro-bellows membrane.

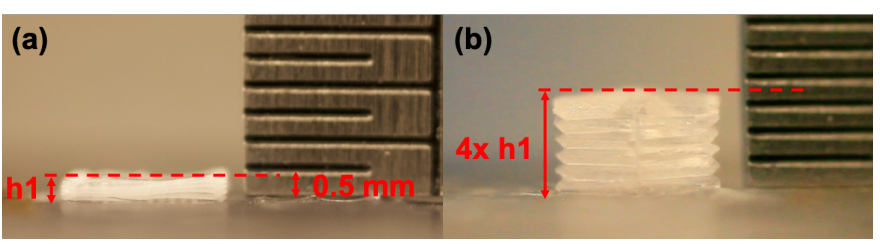

Fig. 6. (a) and (b) Photographs of Parylene $\mathrm{C}$ membrane expansion before and after wireless powering.

remove the unpolymerized excess of resin and washed with isopropyl alcohol. These two steps were repeated until the photoresist was completely removed. Then, the structure was dried with a gentle stream of nitrogen gas.

\section{EXPERIMENTS AND RESULTS}

\section{A. FEM modeling of Parylene C micro-bellows membrane}

Finite element modeling (FEM) analysis was applied to determine the optimal corrugation length and depth distances (Fig. 5a) of the triangular bellows shape that result in the maximum deflection of the membrane. In addition, the mechanical resistance and stress distribution of the Parylene $\mathrm{C}$ micro-bellows membrane was investigated (Fig. 5b). Using Solidworks software (Solidworks 2017, Dassault Systèmes SolidWorks Corporation, MA, USA), a $10 \mu \mathrm{m}$ thick membrane design was simulated in a nonlinear static mode. The optimal corrugation length and depth parameters giving the largest deflection and lowest stress were $250 \mu \mathrm{m}$ respectively.

\section{B. Electrolysis pump assembly and test}

The Parylene $\mathrm{C}$ membrane was assembled on top of the

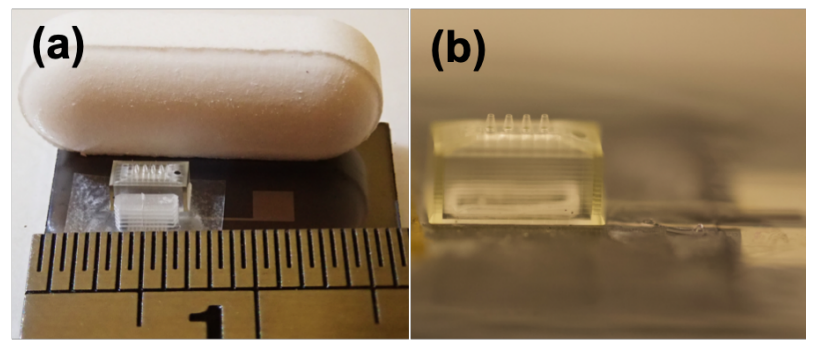

(c)

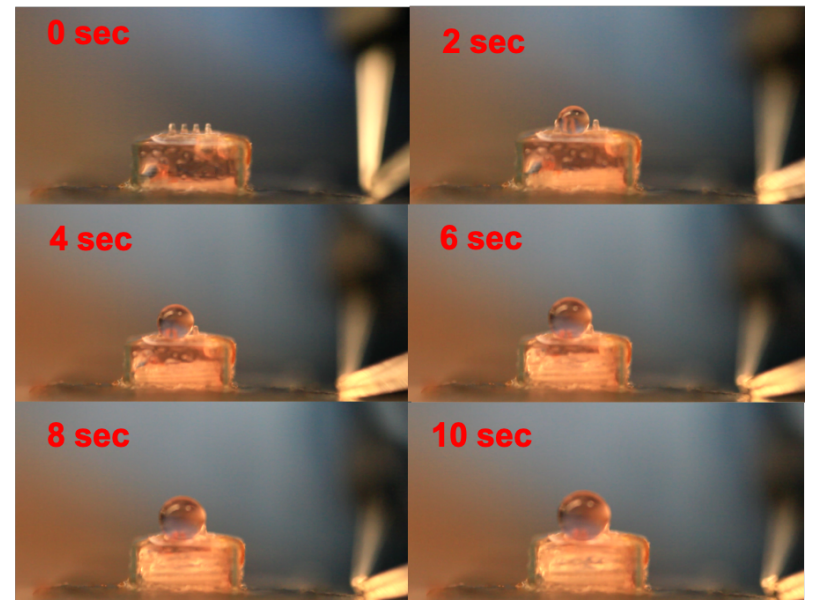

Fig. 7. (a) Photograph of the delivery system components compared to a commercial $500 \mathrm{mg}$ paracetamol tablet. (b) Photograph of the assembled delivery system. (c) Photographs of the dye delivery through micro needles using inductive wireless powering. 
interdigitated electrodes using a Loctite 4011 instant adhesive (Loctite 4011, Henkel, Düsseldorf, Germany). The two holes on the backside of the silicon substrate allowed to inject the electrolyte solution ( $1 \mathrm{wt} \% \mathrm{NaCl}$ solution in DI water) and purge the membrane from any trapped air bubbles, then the holes were sealed with Kapton tape. First, the electrochemical pump was tested by connecting a source meter (Keithley 2400 SourceMeter SMU Instruments, Tektronix, OR, USA). The applied voltage was set to 5 Volts, and the measured power consumption was $25 \mathrm{~mW}$. The micro-bellows membrane expansion reached $1.85 \mathrm{~mm}$ within 40 seconds (initial height was $0.6 \mathrm{~mm}$ ). Then, inductive wireless powering was used with a $10 \mathrm{~mm}$ distance between the transmitter and receiver coils. As shown in Fig. 6, The membrane reached $1.9 \mathrm{~mm}$ of total deflection starting from an initial height of $0.5 \mathrm{~mm}$ achieving about $300 \%$ expansion.

\section{Actuator assembly and test}

The 3D printed reservoir was connected to the electrolysis micro-pump using a Loctite 4011 instant glue (Fig. 7a-b). Then, the integrated system was validated by testing the full assembly. The reservoir was filled with red dye for ease of imaging to mimic the drug during the experiment as shown in Fig. 7c. The micro-bellows geometry and expansion determined the maximum volume to be delivered. In this case, for an expansion of $1.2 \pm 0.1 \mathrm{~mm}$, the amount delivered was $3.8 \pm 0.3 \mu \mathrm{L}$. Fig. 7 depicts the delivery of $\sim 3.8 \mu \mathrm{L}$ red dye through the microneedles within 10 seconds of actuation.

\section{CONCLUSION}

Fabrication process, assembly and testing of a miniaturized drug delivery system have been demonstrated. Emphasis was put on minimizing the device dimensions while providing flexibility in the fabrication process and wireless operation. This was achieved by combining a 3D printed reservoir and microneedles, a micro bellow membrane for large displacements and an electrolytic pump for low-power inductive pumping. The overall size of the device $(3.9 \mathrm{~mm} \times$ $2.1 \mathrm{~mm} \times 2 \mathrm{~mm}$ ) makes it suitable for biomedical applications especially localized drug injection inside the body. Successful delivery of about $3.8 \mu \mathrm{L}$ red dye through microneedles was achieved using wireless inductive powering at $10 \mathrm{~mm}$ distance between the transmitter and receiver. This delivery system showed a compromise between the device size, dose volume, power consumption, and design flexibility, comparing to previous works in [6-8] and [10-11].

\section{ACKNOWLEDGMENT}

This work was funded and supported by King Abdullah University of Science and Technology (KAUST).

\section{REFERENCES}

[1] J. Urquhart, "Controlled drug delivery: therapeutic and pharmacological aspects," Journal of internal medicine, vol. 248, no. 5, pp. 357-376, 2000.

[2] L. Cao, S. Mantell, and D. Polla, "Design and simulation of an implantable medical drug delivery system using microelectromechanical systems technology," Sensors and Actuators A: Physical, vol. 94, no. 12, pp. 117-125, 2001.

[3] R. S. Shawgo, A. C. R. Grayson, Y. Li, and M. J. Cima, "BioMEMS for drug delivery," Current Opinion in Solid State and Materials Science, vol. 6, no. 4, pp. 329-334, 2002.

[4] C. R. Neagu, J. G. Gardeniers, M. Elwenspoek, and J. J. Kelly, "An electrochemical microactuator: principle and first results," Journal of Microelectromechanical systems, vol. 5, no. 1, pp. 2-9, 1996.

[5] C. Neagu, H. Jansen, H. Gardeniers, and M. Elwenspoek, "The electrolysis of water: an actuation principle for MEMS with a big opportunity," Mechatronics, vol. 10, no. 4-5, pp. 571-581, 2000.

[6] P.-Y. Li, R. Sheybani, C. A. Gutierrez, J. T. Kuo, and E. Meng, "A parylene bellows electrochemical actuator," Journal of Microelectromechanical Systems, vol. 19, no. 1, pp. 215-228, 2010.

[7] E. Meng and T. Hoang, "MEMS-enabled implantable drug infusion pumps for laboratory animal research, preclinical, and clinical applications," Advanced drug delivery reviews, vol. 64, no. 14, pp. 1628-1638, 2012.

[8] R. Sheybani, A. Cobo, and E. Meng, "Wireless programmable electrochemical drug delivery micropump with fully integrated electrochemical dosing sensors," Biomedical microdevices, vol. 17, no. 4, p. 74, 2015.

[9] K. Moussi and J. Kosel, "3-D Printed Biocompatible Micro-Bellows Membranes," J. Microelectromech. Syst., vol.27, no.3, pp. 472-478, 2018.

[10] Y. Yi, J. Kosel, “A remotely operated drug delivery system with dose control," Sensors and Actuators A: Physical, vol. 261, pp. 177-183, 2017.

[11] Y. Yi, A. Zaher, O. Yassine, U. Buttner, J. Kosel, and I. G. Foulds, "Electromagnetically powered electrolytic pump and thermo-responsive valve for drug delivery," in 10th IEEE International Conference on Nano/Micro Engineered and Molecular Systems, 2015, pp. 5-8: IEEE.

[12] J. Giannatsis and V. Dedoussis, "Additive fabrication technologies applied to medicine and health care: a review," The International Journal of Advanced Manufacturing Technology, vol. 40, no. 1-2, pp. 116-127, 2009.

[13] W. D. Paulson et al., "Safety and efficacy of local periadventitial delivery of sirolimus for improving hemodialysis graft patency: first human experience with a sirolimus-eluting collagen membrane (CollR)," Nephrology Dialysis Transplantation, vol. 27, no. 3, pp. 1219-1224, 2012 .

[14] A. Nisar, N. Afzulpurkar, B. Mahaisavariya, and A. Tuantranont, "MEMS-based micropumps in drug delivery and biomedical applications," Sensors and Actuators B: Chemical, vol. 130, no. 2, pp. 917-942, 2008.

[15] C. G. Cameron and M. S. Freund, "Electrolytic actuators: Alternative, high-performance, material-based devices," Proceedings of the National Academy of Sciences, vol. 99, no. 12, pp. 7827-7831, 2002. 